\title{
AVANCES EN LA CONSERVACIÓN Y OBTENCIÓN DE MATERIAL SELECTO DEL BANCO NACIONAL DE GERMOPLASMA DE Prosopis, CÓRDOBA, ARGENTINA
}

\author{
Joseau, M. J. ${ }^{1}$; FrassonI, J. ${ }^{1} ;$ VERZINO, G. ${ }^{1}$; \\ Rodríguez Reartes, S. ${ }^{1} ;$ Verga, A. ${ }^{2}$ \& López Lauenstein, D. ${ }^{3}$
}

\begin{abstract}
RESUMEN
El objetivo de esta revisión es presentar los avances en la conservación y en la obtención de material selecto del BNGP. El BNGP cuenta en el Banco Pasivo con 1.650 accesiones correspondientes a 1.106 árboles de nueve especies arbóreas de Prosopis y a 8 poblaciones de $P$. alba que se encuentran bajo estudio para constituirse en Áreas Productoras de Semillas (APS) o Rodales Semilleros (RS). Con la información del Banco de Datos desde 1986 y el aporte de investigaciones se crearon 6 RS en proceso de inscripción como Rodal Semillero, 14 APS inscriptas, 17 nuevas APS en la región del Espinal aún en estudio y 3 en el sur- oeste de Córdoba en proceso de inscripción. Los APS y RS logrados, inscriptos en INASE, se constituyen en material base para la obtención de semillas de las categorías "Fuente identificada" y "Seleccionada", respectivamente, disponibles para abastecer a las necesidades de forestación.
\end{abstract}

Palabras clave: rodales semilleros, áreas productoras de semillas, algarrobo.

1.- Silvicultura. Departamento de Producción Vegetal. Facultad de Ciencias Agropecuarias, Universidad Nacional de Córdoba (FCA-UNC). Félix Aldo Marrone 746. Of. 416. (5000) Córdoba. Argentina.

E-mail: jajoseau@agro.unc.edu.ar

2.- Agencia de Extensión Rural La Rioja.Estación Experimental Agropecuaria Chamical. Centro Regional Catamarca-La Rioja.Instituto Nacional de Tecnología Agropecuaria (INTA). Saavedra 2074, Barrio 25 de Mayo Norte. (5300) La Rioja. Argentina. E-mail: anibal.r.verga@gmail.com

3.- Instituto de Fisiología y Recursos Genéticos Vegetales (IFRGV). Centro de Investigaciones Agropecuarias (CIAP). Instituto Nacional de Tecnología Agropecuaria (INTA). Camino 60 Cuadras, km 5,5. X5020ICA. Córdoba. Argentina. E-mail: lopez.diego@inta.gob.ar Manuscrito recibido el 20 de abril de 2020 y aceptado para su publicación el 11 de septiembre de 2020.

E Joseau M.J., Frassoni J., Verzino G., Rodriguez Reartes S., Verga A., Lopez Lauenstein D. Avances en la conservación y obtención de material selecto del banco nacional de germoplasma de Prosopis, Córdoba, Argentina. FAVE - Ciencias Agrarias 20 (1): 265-285. CC BY-NC-SA 4.0 


\begin{abstract}
Advances in conservation and procurement of selected material by the National Germplasm Bank of Prosopis, Córdoba, Argentina

The objective of this review is to present the advances in conservation and procurement of selected material by the National Germplasm Bank of Prosopis (BNGP). The BNGP has 1,650 accessions in the Base Bank corresponding to 1,106 trees of 9 tree species of Prosopis and 8 populations of $P$. alba that are under study to become Seed Producing Areas (APS) or Seed Stands (RS). With the information from the BNGP data bank since 1986 and the contribution of investigations, 6 RS were created in the process of registration as RS, 14 registered APS, 17 new APS in the Espinal region still under study and 3 in the south-west of Cordoba in the registration process. The APS and RS achieved, registered in INASE, are constituted in base material for obtaining seeds of the categories "Identified source" and "Selected", respectively, available to supply the afforestation needs.
\end{abstract}

Key words: seeds stands, seed zones, “algarrobo".

\section{INTRODUCCION}

Los recursos genéticos forestales (RGF) son el material hereditario que se encuentra dentro y entre las especies de plantas leñosas y árboles, que tienen un valor social, científico, ambiental o económico real o potencial (FAO, 2014b; Mallén Rivera, 2014). Los RGF son esenciales para la adaptación y protección de nuestros ecosistemas, paisajes y sistemas de producción, sin embargo, se encuentran sujetos a una utilización insostenible y a crecientes presiones antrópicas (FAO, 2014b), y ambientales en el marco de los efectos combinados del cambio climático (FAO, 2014b). Sobre la base de la información y conocimientos recopilados por la FAO (2014a) se identificaron 27 prioridades estratégicas, entre ellas, mejorar el uso sostenible y la gestión de los RGF, desarrollando y reforzando los programas de investigación sobre la mejora genética de árboles, domesticación y prospección bioló- gica, como así también desarrollar estrategias nacionales para la conservación in situ y ex situ (FAO, 2014 a y b).

La conservación, entendida como una disciplina dedicada a la protección, rescate, mantención, estudio, intercambio y uso sustentable del patrimonio biológico de un país, es vital para mantener la diversidad genética de especies de este o de una región, así como sus interacciones y los procesos evolutivos que las originan. La conservación de RGF se puede practicar bajo dos modalidades; in situ, conservación dinámica, es decir en el lugar donde los organismos a conservar crecen en estado natural o silvestre, o ex situ, conservación estática, fuera del lugar natural, como es el caso de los bancos de germoplasma y rodales plantados (Salazar et al., 2006, Ipinza et al., 2011, López Lauenstein et al. 2016; Verzino et al., 2019). Ambas opciones son complementarias para la conservación de especies y de su variación genética intraespecífica. 
La conservación dinámica (in situ) busca que la variación genética existente en el material conservado no sólo garantice su adaptabilidad actual, sino también la evolución continua de la especie, manteniendo una amplia diversidad genética y potencial adaptativo, mediante la presión selectiva natural del medio y de la reproducción sexual. De este modo, van apareciendo nuevos genotipos por recombinación y apareamiento, sin llevarse a cabo una selección fenotípica de los árboles progenitores. Esta estrategia requiere una protección efectiva frente a la introgresión genética desde el exterior, así como frente a incendios u otro tipo de amenazas sobre la población conservada. La conservación estática (ex situ) de recursos genéticos considera el principio de preservar la diversidad existente y está ligada a la creación de colecciones que mantienen composiciones genéticas específicas, sin respuesta a posibles cambios en el ambiente, y sin generar nueva diversidad. Los métodos de conservación estática se aplican a un material genéticamente bien identificado y que estabiliza su composición genética actual, definida por el material incorporado a la colección o banco de genes en forma de propágulos por un largo plazo (Ipinza et al., 2011).

Debido a la velocidad del cambio climático y la relativamente lenta respuesta adaptativa de los bosques, la conservación in situ debe ser complementada con otros sistemas cómo lo es el sistema de mejora de poblaciones múltiples (MPBS), desarrollado por Namkoong (1976, 1974), y la conservación ex situ de los RGF, de manera de enfrentar los cambios eco-climáticos con una amplia varianza genética, necesarios además para realizar mejoramiento genético forestal en el futuro (Ipinza et al., 2011,
López Lauenstein et al., 2016) como lo sugiere la FAO (2014b).

Los programas de mejoramiento genético de especies forestales de amplio uso en la silvicultura mundial, como en los géneros Pinus y Eucalyptus, (Castillo Ibáñez y Moreno Días, 2000; FAO, 2014a; Mallén Rivera, 2014) se llevan a cabo con mayor facilidad gracias al cúmulo de conocimientos alcanzados a través de varias décadas de investigación. Sin embargo, en especies nativas poco domesticadas, se ve dificultado un desarrollo fluido de programas de mejoramiento (Castillo Ibáñez y Moreno Días, 2000; FAO, 2014a). Se considera, por lo general, que el objetivo de un programa de mejoramiento genético es el abastecimiento sostenido de semillas y de otros propágulos de alto valor genético que pueden tener fines diferentes, ya sean productivos o de conservación de la variabilidad genética (Castillo Ibáñez y Moreno Días, 2000; Verga, 2014, López Lauenstein, 2016). Por otro lado, el alto valor en las especies nativas no se reduce solamente a la calidad de la madera, sino que se amplía a productos forestales no madereros y a servicios ecosistémicos y ambientales. Muchas de ellas poseen además un alto potencial adaptativo, inclusive con posibilidades de adecuarse a cambios climáticos futuros (Ipinza et al., 2011).

El MPBS, que combina la conservación y la mejora genética, es el mejor método de dirigir los programas de conservación. (Eriksson, Namkoong y Roberds, 1995). Ejemplo del sistema de MPBS aplicado en especies nativas de Argentina, fue el realizado por Verga (2017) para la especie Prosopis alba Grisebach, "algarrobo blanco”, al establecer tres ensayos de progenies en ambientes contrastantes dentro de Argen- 
tina en su área de distribución con aproximadamente 200 familias comunes. En función de los valores de mejora obtenidos del análisis de los tres ensayos, se realizó un raleo genético en uno de ellos, teniendo en cuenta su ranking y también valorando las familias por su plasticidad a través de los rankings de los dos restantes. El raleo se realizó manteniendo todas las familias, eliminando un número variable de individuos por familia según su valor de mejora. De esta forma las familias peor rankeadas quedaron con uno o dos individuos, mientras que las mejores con la totalidad de sus individuos (entre 14 y 15), de esta manera se busca obtener semillas de alta diversidad con un cierto grado de mejora para cada ambiente (Verga 2017).

El género Prosopis posee 44 especies y 27 variedades (Burkart, 1976). La existencia de poblaciones de Prosopis con características diferentes condujo a Burkart (1976) a describir a algunas como especies separadas o variedades, incluso varias son conocidas como híbridos (Pasiecznik et al., 2001; Joseau et al., 2013a; López Lauenstein et al., 2016). Algunas especies de la Sección Algarobia (P. alba, P. nigra (Grisebach) Hieronymus "algarrobo negro", "árbol negro”, P. chilensis (Mol.) Stuntz, "algarrobo blanco", "algarrobo de Chile”, "árbol blanco" у P. flexuosa DC., "algarrobo negro", "algarrobo dulce", "árbol negro") tienen capacidad de hibridarse en forma frecuente u ocasional, razón por la cual diversos autores han sugerido que las mismas constituyen un singámeon (Palacios et al., 1988; Saidman, 1986; Bessega, 1997), definido por Grant (1981) como un grupo de especies que hibridan y se comportan como una especie biológica, aislada reproductivamente de otros grupos similares.
Argentina posee 27 especies de Prosopis y 21 de ellas pertenecen a la sección Algarobia (Palacios et al., 1988), sección dónde se destacan por su porte arbóreo nueve especies que son las que fueron seleccionadas como prioritarias para la conservación de la variabilidad genética existente y comercialización por el Banco Nacional de Germoplasma de Prosopis de la Facultad de Ciencias Agropecuarias de la Universidad Nacional de Córdoba (BNGP-FCA-UNC) desde su formación en el año 1985.

El objetivo de esta revisión es presentar los avances en la conservación y en la obtención de material selecto del Banco Nacional de Germoplasma de Prosopis (BNGP).

\section{DESARROLLO}

\section{Proyección nacional del BNGP}

El BNGP conserva, actualmente, en su banco base (Banco Pasivo), 1.650 accesiones, correspondientes a 1.106 árboles y 8 poblaciones de $P$. alba que se encuentran bajo estudio para constituirse en APS o RS. Las semillas individualizadas por accesión, previamente secadas en estufa hasta $6 \%$ de humedad, se conservan desde su colecta en freezer a $-18{ }^{\circ} \mathrm{C}$. Los primeros materiales fueron mantenidos en envase de plástico con rosca y silica gel y desde el año 2016, todas las accesiones se transvasaron a pouches trilaminados (Poliester + Aluminio + Polietileno de $110 \mathrm{~mm}$ x $150 \mathrm{~mm}$ ) con capacidad de $60 \mathrm{~g}$ (aproximadamente 1.000 semillas), sellados con máquina selladoras de pouches (Verzino et al., 2020). 
Los frutos de Prosopis del que se obtuvieron las semillas se colectaron de árboles individuales distanciados a más de 100 cien metros de otro ejemplar. Este aislamiento se efectuó a los fines de evitar depresión por endogamia (Verzino et al., 2005; Bessega et al., 2012), lo que ha permitido que la semilla sea aceptada como material básico tal cual lo establece la Res. 374/14 del INASE (INFOLEG, 2019).

El BNGP se encuentra inscripto en el Registro Nacional del Comercio y Fiscalización de Semillas del INASE (Instituto Nacional de Semillas) con el $\mathrm{N}^{\circ}$ 1559ACHIJ1K1. Esta inscripción permite comercializar semillas de origen y calidad (Banco Activo), semillas que se almacenan en envases plásticos a rosca, herméticos, con una bolsa que contiene sílice gel para mantener la humedad entre 6-9 \% en condiciones de freezer a $-18{ }^{\circ} \mathrm{C}$. Entre ellas se destacan $P$. alba, $P$. nigra, $P$. flexuosa, $P$. chilensis y $P$. caldenia Burkart, "caldén. Otras especies arbóreas que forman parte del Banco Pasivo son $P$. ferox Grisebach "churqui”, “churqui blanco", $P$. hassleri Harms "algarrobo paraguayo”, P. kuntzei Harms "itín”, "palo mataco", "barba de tigre”, y P. affinis Grisebach Sprengel, “ñandubay”, “algarrobillo”.

La recolección del material seminal que se conserva actualmente en el Banco Pasivo se efectuó con fondos propios o mediante proyectos de Investigación financiados por FAO-Montes, Academia Nacional de Ciencias de EEUU (NAS), CAFPTA, CONICET-SECYT, SECYT-UNC, SECYT-FCA, INTA, Proyecto Forestal de Desarrollo y PIA de SAGPyA, Banco Mundial, PID y PIODO de MINCYT-Córdoba, entre otros. El material procedió de 14 provincias (Catamarca, Chaco, Córdoba, Entre Ríos, Formosa, Jujuy, La Pampa, La Rioja, Salta,
San Juan, San Luis, Santa Fe, Santiago del Estero, Tucumán). Los miembros de la Comisión Nacional de Prosopis (1985-2003) contribuyeron a realizar los lazos entre las entidades que participaron en las primeras cosechas e investigaciones. Participaron en la recolección aproximadamente 110 personas entre estudiantes, técnicos y profesionales en la recolección pertenecientes a diversos organismos que colaboraron en la recolección del material. Se destacan en Córdoba, la FCA-UNC, la Estación Forestal INTA Villa Dolores y el CIAP-INTA. Por Mendoza se contó con la participación del IADIZA (Instituto Argentino de Investigaciones de Zonas Áridas). Además contribuyeron en la colecta reparticiones del INTA ubicadas en Chaco, Entre Ríos, Formosa, Jujuy, La Rioja, Mendoza, Salta, Santiago del Estero y Tucumán. También hubo participantes de la Universidad $\mathrm{Na}$ cional de Formosa y de las Universidades Nacional y Católica ambas de Santiago del Estero. Como organismos gubernamentales se contó con colaboración de la Dirección de Bosques de la provincia de Jujuy, el gobierno de Formosa y personal del Ministerio de Agricultura, Ganadería y Pesca de la Nación. Actualmente, los frutos se compran o son donadas por organismos públicos o privados inscriptos en INASE bajo la categoría Áreas Productoras de Semillas como El Resurgir del algarrobal, S.A. de Catamarca; San Miguel y Villa María de la provincia de Córdoba.

El accionar del BNGP responde al Convenio sobre Diversidad Biológica de la Conferencia de las Partes (www.biodiv.org) y a la Estrategia Nacional sobre Diversidad Biológica de la República Argentina, aprobada por Resolución 91/03 (SAyDS, 2003). El BNGP-FCA-UNC cumple sus funciones 
de acuerdo con el Código Internacional de conducta para la recolección y transferencia de germoplasma vegetal (FAO 1994) así como las Normas Internacionales para Bancos de Genes (FAO/IPGRI 1994, Verzino y Joseau, 2005). Estos convenios internacionales regulan el acceso, la transferencia segura y los derechos y responsabilidades de las partes con respecto a la utilización del germoplasma transferido (Verzino y Joseau, 2005).

El BNGP-FCA-UNC en sus comienzos tenía como objetivo conservar la variabilidad existente sin tener en cuenta la hibridación interespecífica, frecuente en el género, por lo que el banco activo comercializaba semilla de "Fuente identificada" (categoría mínima admitida con respecto a la cual únicamente se tiene información sobre la situación geográfica del área o región donde la semilla es recolectada). Fue hacia el año 1999, con los avances científicos obtenidos en la tesis del Dr. Verga (1995) que se comienzan a seleccionar individuos de poblaciones de $P$. chilensis aislados respecto de otras especies afines, de manera que la probabilidad de tener descendencia híbrida fuese lo más baja posible y de esta manera abastecer a viveros y/o productores con semilla más homogénea y probablemente más estable desde el punto de vista genético para la producción de plantines para plantaciones forestales.

En el año 2005, por iniciativa del Ministerio de Agricultura, Ganadería y Pesca de la Nación, en convenio con el INTA, surgió el Programa Nacional de Mejoramiento de Especies Forestales y a través del Sub programa Prosopis se implementaron investigaciones orientadas a la obtención de información para lograr estrategias guiadas tanto a la conservación in situ, como ex situ y a emprender programas de mejora genética de un género con amplia variabilidad genética y con presencia de hibridación (Marcó et al. 2016, López Lauenstein et al., 2016). Muchas de dichas investigaciones encontraron en el BNGP un lugar para la conservación ex situ de las semillas y/o herbarios recolectados bajo el nombre de colección INTA, mientras que otras fueron introducidas a plantaciones en forma de ensayos de progenies, orígenes y poblaciones múltiples (Joseau y Verzino, 2013,Verzino et al., 2019, Joseau y Verzino, 2013, Verga, 2017).

Paralelamente, la ley $\mathrm{N}^{\circ} 25.080$ tuvo como exigencia que para poder acceder al sistema de promoción forestal era necesario utilizar material inscripto en el INASE. La Resolución N 256/99 del INASE fijó las normas para la certificación, producción, comercialización e importación de semillas de especies forestales (INASE, 2019a). Asimismo, en el Artículo $4^{\circ}$ establece que el material básico (árboles o partes de plantas de los cuales se obtiene material reproductivo) puede obtenerse de diversas formaciones, entre ellas los Rodales Semilleros (RS) siendo definido como: "grupo de árboles de base genética amplia, lo suficientemente aislado para reducir los efectos contaminantes de polen foráneo, y que por presentar características deseables en cuanto a forma, crecimiento y sanidad de los árboles, es manejado para producir semilla. Aquí los individuos de baja calidad se eliminan y solamente se conservan los mejores progenitores para cruzarlos. Estos son seleccionados solamente por sus cualidades fenotípicas y rara vez son sometidos a pruebas de progenies. Se conoce el origen o procedencia de los árboles progenitores"; y las Áreas Productoras de Semillas (APS): "el espacio físico de producción de 
semilla que no cumple con uno o más requisitos de la definición anterior o de otros materiales bases de mayor jerarquía” (Joseau et al. 2013a).

A fin de promover el uso de materiales de identidad reconocida se aprobó la Resolución 102/2010 de la Secretaría de Agricultura, Ganadería, Pesca y Alimentos de la Nación Argentina, que establece un incremento en el pago del apoyo económico instituido por la Ley $\mathrm{N}^{\circ} 25.080$ en un diez por ciento (10 \%) para las actividades de plantación y enriquecimiento del bosque nativo con especies nativas y exóticas de alto valor comercial, cuando se trata de material certificado (Joseau et al., 2013a).

Ante el riesgo de pérdida de biodiversidad en Argentina, dada por situaciones de cambio climático y pérdida de bosques, que ha ocurrido durante estos últimos 35 años, concentrada principalmente en la Región Chaqueña (Rocha, 2018), se delimitaron APS para ser transformadas en lo posible en RS a fin de preservar la variabilidad existente y obtener material base con mayor uniformidad genética y llegar a obtener una categoría certificada dada por INASE. De esa manera se supera el inconveniente que procede del intercambio genético entre especies que se da naturalmente en este complejo (Verga, 2014), dando respuesta a la Res.102/2010 y a las sugerencias de la FAO (2014) relativos a mejorar el uso sostenible y la gestión de los RGF.

Los conocimientos genéticos y ecofisiológicos obtenidos sobre $P$. chilensis, $P$. flexuosa y P. alba (Verga et al., 2009; Verga et al., 2014; Joseau et al., 2013b, López Lauenstein et al., 2013, Ferreyra et al., 2013) constituyeron la base conceptual para avanzar en el mejoramiento y conservación de los RGF de Prosopis. El BNGP a través de un esfuerzo conjunto con el CIAP-INTA, la Agencia de Extensión Rural Andalgalá del INTA, Esta- ción Experimenta Agropecuaria Yuto del INTA, la Regional Córdoba y la Regional NOA de la Dirección de Producción Forestal (DPF) del MAGyP de la Nación desarrolló el proyecto denominado "Fortalecimiento del Banco Nacional de Germoplasma de Prosopis para la conservación in situ y ex situ de $P$. alba Grisebach, $P$. flexuosa DC., $P$. chilensis (Mol.) Stuntz y P. caldenia Burkart y la obtención de semillas con destino a plantaciones forestales de calidad" (Proyecto Silva N³4. GEF090118) para, en primer lugar, consolidar su funcionamiento como organismo de conservación de germoplasma del género Prosopis y, en segundo lugar, obtener semilla de $P$. alba y $P$. flexuosa, $P$. chilensis de categoría "Seleccionada".

\section{Tipos de materiales básicos obtenidos por} el BNGP

Categoría: "Fuente identificada" Delimitación e inscripción de Áreas Productoras de Semillas (APS). La fructificación del algarrobo está sujeta a variaciones cíclicas, es decir, no hay disponibilidad estable de semillas año a año. Por esta razón, es importante no depender de una única fuente semillera para poder abastecerse de semillas periódicamente. Si bien la disponibilidad de información respecto al comportamiento del material genético a utilizar en las plantaciones es insuficiente porque no existe material probado en ensayos para la gran variabilidad de ambientes posibles, se han realizado experiencias locales como los citados por Joseau et al. (2012), Verzino y Joseau (2013), Verzino et al. (2019). Por otro lado se desconoce el impacto que tendrá la introducción de material genético de una zona a otra (Zarate et al., 2016).

Teniendo en cuenta los materiales y datos existentes en la Base de Datos del BNGP-FCA-UNC, que data desde 1986, y 


\section{J. Joseau et al.}

nuevos aportes investigativos, se han regis- obtención anual de frutos.

trado en INASE hasta el momento 14 APS en la provincia de Córdoba, compuestas por 6 poblaciones de $P$. alba, 4 de $P$. chilensis, 2 de $P$. flexuosa, 1 de P.chilensis x P. flexuosa y 1 de Prosopis sp., dando origen a un número de identificación de Material Básico Forestal por población que se presenta en la Tabla 1. Existen más poblaciones, pero por razones presupuestarias aún falta incorporarlas al INASE como nuevas APS. Se cuenta para la obtención de material, desde la generación de estos registros, con el permiso de la Secretaria de Ambiente y Cambio Climático del Ministerio de Servicios Públicos de la Provincia de Córdoba para la
Asimismo, para obtener respuestas a la problemática del desempeño de las distintas procedencias en plantaciones fuera de su rango de distribución, y específicamente para la Provincia de Córdoba, surgió en el año 2015 la tesis doctoral del Ing. Agr. Javier Frassoni en el marco del BNGP denominada: "Diversidad adaptativa y morfológica de poblaciones progenies de P. alba Grisebach. frente a diversos escenarios de cambio climático en el Parque Chaqueño argentino y el Espinal”, que amplía la exploración del territorio nacional en la búsqueda e identificación de nuevas APS en la región fitogeográfica del Espinal.

Tabla 1. Áreas Productoras de Semillas de la provincia de Córdoba inscriptas por el BNGP o con la participación del BNGP en la Categoría de Fuente Identificada.

Table 1. Seed Production Areas of Córdoba province registered by the BNGP or with the participation of the BNGP in the Identified Source Category

\begin{tabular}{|c|c|c|c|}
\hline Especie & $\begin{array}{c}\mathrm{N}^{\circ} \text { de Material Básico } \\
\text { Forestal }\end{array}$ & Localización & Razón Social \\
\hline P. alba & $8 \times 5527 \mathrm{JPr}$ & Córdoba Capital & UNC-BNGP \\
\hline P. alba & 9X5527JPr & Córdoba Capital & UNC-BNGP \\
\hline P. alba & $10 \times 5527 \mathrm{JPr}$ & Piquillín & UNC-BNGP \\
\hline P. alba & 11X5527JPr & Tránsito & UNC-BNGP \\
\hline P. alba & 12X5527JPr & Villa del Rosario & UNC-BNGP \\
\hline P. chilensis & $3 \times 5527 \mathrm{JPr}$ & El Portezuelo & UNC-BNGP \\
\hline P. chilensis & $4 \times 5527 \mathrm{JPr}$ & Chancaní & UNC-BNGP \\
\hline P. chilensis & $5 \times 5527 \mathrm{JPr}$ & $\begin{array}{l}\text { Villa de Soto, } \\
\text { Serrezuela }\end{array}$ & UNC-BNGP \\
\hline P. chilensis & $6 \times 5527 \mathrm{JPr}$ & $\begin{array}{l}\text { Cruz del Eje, } \\
\text { Media Naranja }\end{array}$ & UNC-BNGP \\
\hline P. chilensis $x$ P.flexuosa & 7X5527JPr & $\begin{array}{l}\text { Charbonier, Las } \\
\text { Escobas }\end{array}$ & UNC-BNGP \\
\hline P. flexuosa & 1X5527JPr & San Miguel & UNC-BNGP \\
\hline P. flexuosa & 2X5527JPr & El Portezuelo & UNC-BNGP \\
\hline P. alba & $13 \times 10034 J P r$ & Villa María & Destefanis Osvaldo Ignacio \\
\hline P. sp. & 14X5527JPr & Tio Pujio & UNC-BNGP \\
\hline
\end{tabular}


En dicha tesis se han identificado 22 posibles poblaciones de $P$. alba como fuentes de semillas de categoría identificada, georreferenciando 241 árboles en total. Los árboles muestreados estaban separados más 100 metros uno de otros siguiendo las especificaciones de Verzino et al. (2005) y Bessega et al. (2012). Se extrajeron muestras de suelo y hojas para realizar una caracterización edáfica y morfológica respectivamente. La caracterización edáfica fue realizada a través de la determinación de $\mathrm{pH}$, conductividad eléctrica, color, y textura de todos los horizontes muestreados, y de materia orgánica del horizonte superficial; mientras que la caracterización morfológica se llevó a cabo siguiendo el protocolo sugerido por Verga (2005), Joseau et al. (2005), Joseau (2006) y Joseau et al. (2013), utilizando para esto el programa Hojas 3.4. (Verga, 2000). La Tabla 2 presenta la denominación de las poblaciones y detalles del material colectado. El material recolectado fue incorporado a la base de datos del BNGP y cinco de los sitios: Piquillín, Tránsito, Villa del Rosario, Plaza de los algarrobos y Parque de la Vida (Córdoba Capital) fueron inscriptos como APS (Tabla 1).

Tabla 2. Denominación de las poblaciones, cantidad de árboles y material colectado de P. alba del Espinal.

Table 2. Denomination of populations, number of trees and material collected of Espinal P. alba.

\begin{tabular}{|c|c|c|c|c|c|c|}
\hline \multirow[t]{2}{*}{ Población } & \multirow[t]{2}{*}{ Denominación } & \multirow[t]{2}{*}{ Provincia } & \multirow{2}{*}{$\begin{array}{l}\text { Fecha de } \\
\text { recolección }\end{array}$} & \multicolumn{3}{|c|}{ Árboles } \\
\hline & & & & Cantidad & Con hojas & Con frutos \\
\hline 1 & Monte Cristo & Córdoba & \multirow{8}{*}{ Ene. 2016} & 10 & 10 & 6 \\
\hline 2 & Piquillín & Córdoba & & 3 & 3 & 2 \\
\hline 3 & Río I & Córdoba & & 11 & 11 & 10 \\
\hline 4 & Los Chañaritos & Córdoba & & 10 & 10 & 3 \\
\hline 5 & La Francia & Córdoba & & 11 & 11 & 2 \\
\hline 6 & Parque San Martín & Entre Ríos & & 35 & 29 & 7 \\
\hline 7 & Colonia Nueva & Santa Fe & & 10 & 10 & 3 \\
\hline 8 & Don Justiniano & Córdoba & & 12 & 12 & 4 \\
\hline 9 & Plaza de los Algarrobos & Córdoba & \multirow{14}{*}{ Feb. 2017} & 13 & 13 & 10 \\
\hline 10 & Parque de la Vida & Córdoba & & 13 & 13 & 12 \\
\hline 11 & Universidad Católica & Córdoba & & 10 & 10 & 0 \\
\hline 12 & Este de RP 3 & Córdoba & & 10 & 9 & 0 \\
\hline 13 & Balnearia & Córdoba & & 10 & 10 & 0 \\
\hline 14 & Norte en RP 70 & Santa Fe & & 12 & 6 & 0 \\
\hline 15 & Esperanza & Santa Fe & & 11 & 11 & 0 \\
\hline 16 & Las Mojarras & Córdoba & & 11 & 11 & 4 \\
\hline 17 & RN 19 & Santa Fe & & 7 & 2 & 0 \\
\hline 18 & Cañada de Machado & Córdoba & & 8 & 8 & 4 \\
\hline 19 & Villa del Rosario & Córdoba & & 11 & 11 & 8 \\
\hline 20 & Ballesteros & Córdoba & & 10 & 10 & 6 \\
\hline 21 & Belle Ville & Córdoba & & 10 & 10 & 5 \\
\hline \multirow[t]{2}{*}{22} & Sur en RP 2 & Córdoba & & 10 & 10 & 5 \\
\hline & Total & & & 241 & 228 & 91 \\
\hline
\end{tabular}




\section{J. Joseau et al.}

Algunas APS establecidas por el BNGP han avanzado o están en proceso de ser RS (Tabla 5). En enero de 2020, se establecieron 3 APS más hacia el sur-oeste de Córdoba, llegando al límite con San Luis. Cuenta con ejemplares cuyas edades oscilan entre 150 a 370 años, y se encuentra aún en evaluación. Es de destacar que la cosecha de enero del año 2020 ha sido excepcional en la producción de frutos de esta región.

La Figura 1 muestra imágenes de los árboles de $P$. chilensis colectados en 2018 para la formación del APS de Media Naranja- Cruz del Eje y también frutos de $P$. flexuosa colectados de Pipanaco.

\section{Categoría: "Seleccionada"}

Conformación de Rodales Semilleros.

El material de partida para la obtención de semillas de la categoría Seleccionada fueron poblaciones con predominancia de las especies $P$. flexuosa, $P$. chilensis o $P$. alba ubicadas en sus áreas de distribución en las provincias de Catamarca, Córdoba y Salta. Estas

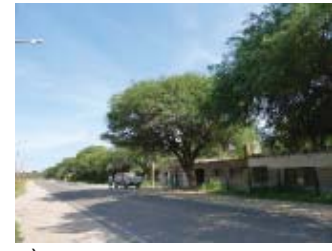

a)

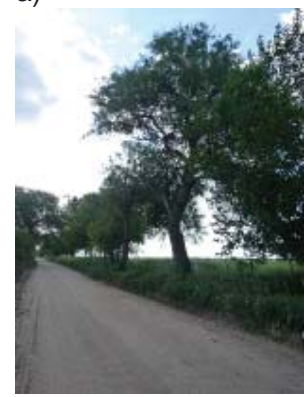

d)

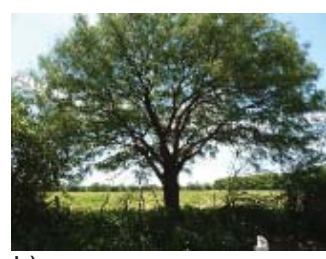

b)

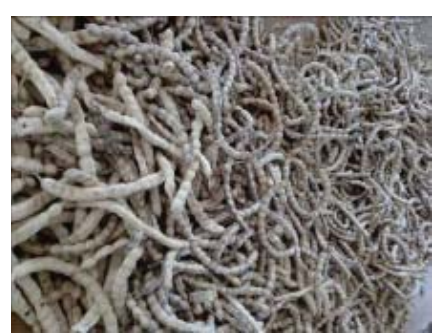

e)

c)

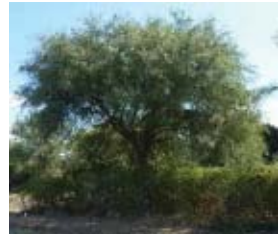$$
\text { . }
$$

Figura 1. Imágenes ( $a, b, c$ y d) de ejemplares de P. chilensis componentes del APS6X5527JPr. Media Naranja-Cruz del Eje; e) Frutos de P. flexuosa (cosecha 2018) procedentes del Rodal Pipanaco (Catamarca) con inscripción en trámite por la empresa Resurgir del Algarrobal S.R.L. Figure 1. Images ( $a, b, c$ and d) of specimens of P. chilensis, components of the APS6X5527JPr. Media Naranja-Cruz del Eje; e) Fruits of P. flexuosa (2018 harvest) from the Pipanaco Stand (Catamarca) with pending registration by the company Resurgir del Algarrobal S.R.L. 
poblaciones fueron detectadas y localizadas a partir de información disponible en las bases de datos del BNGP e INTA, referencias bibliográficas, informantes locales e imágenes satelitales de Google Earth. Se realizaron viajes exploratorios para su confirmación y fueron seleccionados teniendo en cuenta varios aspectos: 1 . Composición específica del rodal, con predominancia de la especie objetivo; 2. Características morfológicas y sanitarias de los árboles; 3 . Accesibilidad del rodal y 4. Condiciones legales del establecimiento donde está ubicado el rodal y predisposición de sus propietarios u ocupantes.

Las poblaciones seleccionadas se constituyeron en APS, ubicados en campos privados o fiscales alambrados y para ser transformadas en RS fueron muestreadas siguiendo la metodología recomendada por Verga (2014) que es la que da origen a la Resolución 374/14 del INASE (2019b). Ésta consistió en un monitoreo mediante la enzima Alcohol Deshidrogenasa (ADH) y la realización de un raleo genético en función de la clasificación de cada uno de los individuos involucrados y de su entorno, mediante un análisis morfológico realizado a partir de muestras de hojas y frutos. El análisis isoen- zimático mediante la $\mathrm{ADH}$ de las semillas de una muestra del rodal determina qué proporción proviene de cruzamientos interespecíficos entre algarrobos del grupo de los "blancos": para este estudio serían P. alba, y $P$. chilensis, y del grupo de los "negros": $P$. nigra y $P$. flexuosa (así llamados por la coloración de sus frutos) (Verga, 2014).

Pre-muestreo exploratorio. La cantidad de árboles muestreados durante la primera exploración, osciló entre 6 y 25 individuos, dependiendo de los ejemplares fructificados dentro de las áreas boscosas seleccionadas. Cada árbol se enumeró con pintura, se georeferenció y se fotografió para poder apreciar sus características morfológicas y estimar sus medidas alométricas. Se colectaron frutos y hojas para el análisis morfológico y genético y para la confección de herbarios que fueron incorporados al Herbario ACOR de la FCA-UNC.

El análisis morfológico se realizó con una muestra de cinco frutos enteros y sanos (vainas) extraídos de cada árbol, medidos manualmente siguiendo la metodología descripta por Verga (1995) (Figura 2a) y con cinco hojas adultas por árbol las cuales

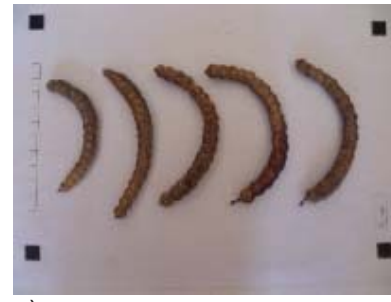

a)

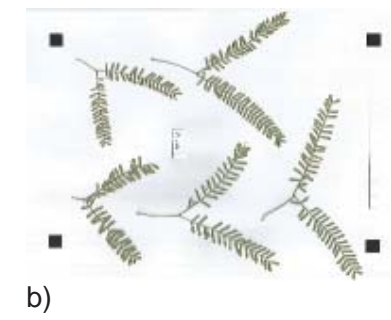

b)

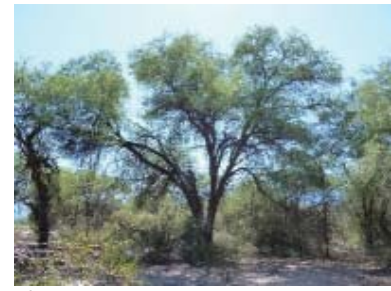

c)

Figura 2. Material utilizado para el análisis morfológico de: a) frutos y b) hojas del APS de P. flexuosa ubicado en Pipanaco, Catamarca. c) Ejemplar de P. flexuosa (Árbol 6). Proyecto Silva N³4. GEF090118.

Figure 2. Material used for the morphological analysis of: a) fruits and b) leaves of the APS of P. flexuosa located in Pipanaco, Catamarca. c) P. flexuosa specimen (Tree 6). Silva Project No. 34. GEF090118. 
fueron escaneadas (Figura 2b). Las semillas se obtuvieron mediante trilla manual de las vainas para el análisis genético.

En cada hoja se midieron, ocho caracteres utilizando el Programa Hojas 3.4 (Verga, 2010): Longitud del pecíolo (cm), número de pares de pinas, longitud de la pina $(\mathrm{cm})$, número de pares de foliólulos por pina, distancia entre foliólulos, longitud del foliólulo (cm), ancho del foliólulo (cm), área del foliólulo $\left(\mathrm{cm}^{2}\right)$ y se calcularon algunas variables como la relación longitud/ ancho de foliólulo, relación área del ápice del foliólulo/área total del foliólulo e índice de área total de la hoja. En tanto, las variables del fruto: longitud, ancho, grosor, forma, color y borde, fueron medidas siguiendo las recomendaciones de Verga (1995) y Joseau et al. (2013b).

En laboratorio se evaluó la pureza específica de la semilla mediante el marcador ADH para $P$. alba, $P$. chilensis y $P$. flexuosa. En cada corrida electroforética se utilizaron 60 semillas, generalmente tres semillas por árbol, conservando la identidad de cada árbol. En la Figura 3a se muestran los resultados para $P$. flexuosa de Pipanaco después del raleo genético y en la Figura $3 \mathrm{~b}$ para $P$. alba previo al raleo genético.

Los análisis isoenzimáticos y los análisis multivariados de Conglomerados y de Componentes Principales) realizados sobre las variables de hojas y frutos fueron efectuados mediante los programas informáticos Infostat (Di Rienzo et al., 2012) y NTSYSpc (Rohlf, 2000). Esto permitió ubicar y delimitar, dentro de cada APS, los sectores de mayor pureza específica para cada especie, los que constituyeron los rodales semilleros propiamente dichos.
Muestreo. Una vez seleccionados y delimitados los rodales, se extendieron los análisis morfológicos de hojas a todos los árboles de cada rodal, incluso aquellos hasta $50 \mathrm{~m}$ por fuera del perímetro. Esta tarea se realizó en dos etapas: en la primera etapa se muestrearon entre 80 y 100 árboles de cada rodal (con excepción de La Unión, donde se muestreó el rodal completo en una etapa, y Palampa, donde el rodal completo quedó conformado por 57 árboles). En la segunda etapa, luego de construir los mapas con la ubicación de los árboles y detectar los vacíos, se muestrearon los árboles restantes (los del perímetro) (Tabla 3).

Se cosecharon frutos de todos los árboles (que hubieran fructificado) para los análisis morfológicos e isoenzimáticos (Figura 3a y b). Mediante análisis multivariado de las variables de hojas (y en algunos casos, de frutos) y con el auxilio de los análisis de $\mathrm{ADH}$, se identificaron los individuos fuera de tipo.

Los raleos silviculturales preliminares, para eliminar algunos ejemplares que no cumplen con las condiciones mínimas de distanciamiento, forma y sanidad quedaron a cargo de los productores (San Miguel P.flexuosa y Villa María - P. alba).

Los análisis morfogenéticos condujeron a efectuar un raleo genético eliminando los árboles fuera de tipo sobre los rodales de Pipanaco, Palampa, La Unión y Villa María, luego se cosecharon frutos de 22 árboles de $P$. chilensis en Palampa y 53 árboles de $P$. flexuosa en Pipanaco para realizar la verificación de la pureza específica. También se cosecharon frutos de los rodales de La Unión y Villa María, cuyo análisis enzimático se encuentra en proceso. 

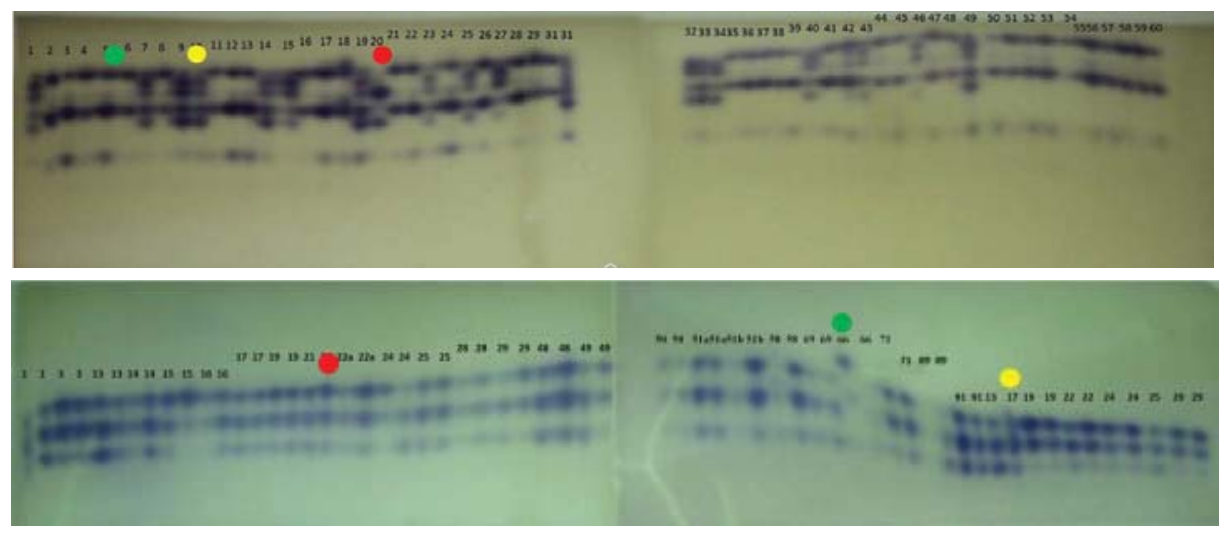

Figura 3. Zimograma electroforético de los loci ADH de una muestra de 60 semillas cosechadas de: a) 53 árboles luego del raleo genético en el rodal de P.flexuosa en Pipanaco. b) 25 árboles de $P$. alba previos al raleo genético de Villa María con frecuencias genotípicas diferentes a las de $P$. flexuosa. El círculo verde representa a los genotipos $A D H-A 22$, el amarillo al genotipo ADH-A23, mientras que la rojo representa a los genotipos ADH-A33 Proyecto Silva N³4. GEF090118. Figure 3. Electrophoretic zymogram of the ADH loci of a sample of 60 seeds harvested from a) 53 trees after genetic thinning in the stand of P.flexuosa in Pipanaco. b) 25 trees of P. alba before genetic thinning in Villa María with genotype frequencies different from those of $P$. flexuosa from " $a$ ". The green circle indicates an individual with the ADH-A22 genotype, the yellow one indicates the ADH-A23 genotype, while the red one represents genotypes that are ADH-A33. Silva Project No. 34. GEF090118 


\section{J. Joseau et al.}

Tabla 3. APS estudiadas para su transformación en rodales semilleros con detalle del número de árboles de cada etapa y análisis realizados.

Table 3. APSs studied for transformation into seed stands with details of the number of trees in each stage and analysis performed.

\begin{tabular}{|c|c|c|c|c|c|c|c|}
\hline RODAL & & & Pipanaco & San Miguel & Palampa & $\begin{array}{c}\text { Villa } \\
\text { María }\end{array}$ & La Unión ${ }^{(1)}$ \\
\hline ESPECIE & & & $\begin{array}{r}\text { P.flexuos } \\
a\end{array}$ & P.flexuosa & P.chilensis & P.alba & P.alba \\
\hline SUPERFICIE DEL & DAL & & 4,3 & 3,9 & 5 & 3 & 2 \\
\hline \multirow{6}{*}{ PRE-MUESTREO } & \multirow{2}{*}{\multicolumn{2}{|c|}{$\begin{array}{l}\text { Cantidad de árboles } \\
\text { Árboles con frutos }\end{array}$}} & 20 & 25 & 10 & 22 & \\
\hline & & & 20 & $25^{*}$ & 6 & 5 & \\
\hline & \multirow{2}{*}{$\begin{array}{l}\text { Análisis } \\
\text { isoenzimá } \\
\text { tico }\end{array}$} & Cantidad & 20 & 25 & 6 & 5 & \\
\hline & & Tipo & Adh & Adh & Adh & Adh & \\
\hline & \multirow{2}{*}{$\begin{array}{l}\text { Análisis } \\
\text { Morfológic } \\
\text { o }\end{array}$} & Hoja & 20 & & 6 & 22 & \\
\hline & & Frutos & 20 & 0 & 6 & 5 & \\
\hline \multirow{6}{*}{ MUESTREO } & \multicolumn{2}{|c|}{ Cantidad de árboles } & 185 & 263 & 57 & 167 & 205 \\
\hline & \multicolumn{2}{|c|}{ Árboles con frutos } & 76 & $\begin{array}{r}\text { Sin } \\
\text { producción }\end{array}$ & 1 & 25 & 15 \\
\hline & \multirow{2}{*}{$\begin{array}{l}\text { Análisis } \\
\text { isoenzimá } \\
\text { tico }\end{array}$} & Cantidad & 60 & 40 & 7 & 25 & 15 \\
\hline & & Tipo & Adh & ------ & Adh & Adh & Adh \\
\hline & \multirow{2}{*}{$\begin{array}{l}\text { Análisis } \\
\text { Morfológic } \\
\text { o }\end{array}$} & Hoja & 175 & 263 & 57 & 167 & 155 \\
\hline & & Frutos & 44 & 0 & 0 & 25 & 13 \\
\hline \multirow[t]{2}{*}{ RALEO } & \multicolumn{2}{|c|}{ Árboles talados } & 7 & & 5 & 3 & 10 \\
\hline & \multicolumn{2}{|c|}{ Árboles con frutos } & 53 & Sin frutos & 22 & Cosecha & Cosecha diciembre \\
\hline \multirow[t]{2}{*}{$\begin{array}{l}\text { ANÁLISIS POS- } \\
\text { RALEO }\end{array}$} & $\begin{array}{l}\text { Análisis } \\
\text { genético }\end{array}$ & $\begin{array}{l}\text { Cantid } \\
\text { ad }\end{array}$ & 53 & $\begin{array}{r}\text { diciembre } \\
2015\end{array}$ & 22 & $\begin{array}{r}\text { Enero } \\
2016\end{array}$ & \\
\hline & & Tipo & Adh & & Adh & & \\
\hline
\end{tabular}

(1) Sin pre-muestreo

Tabla 4. Frecuencia genotípica y alélica del rodal de P.chilensis del establecimiento agrícola Palampa S.A., Catamarca.

Table 4. Genotypic and allelic frequency of the P.chilensis stand of the Palampa S.A. agricultural establishment, Catamarca.

\begin{tabular}{|c|c|c|c|c|c|}
\hline \multicolumn{6}{|c|}{ Frecuencia Genotípica } \\
\hline \multicolumn{3}{|c|}{ Antes del raleo genético } & \multicolumn{3}{|c|}{ Después del raleo genético } \\
\hline Genotipo & Frec.Abs. & Frec.Rel. & Genotipo & Frec.Abs. & Frec.Rel. \\
\hline 33 & 40 & 95,24 & 33 & 59 & 98,33 \\
\hline 23 & 1 & 2,38 & 23 & 1 & 1,67 \\
\hline 22 & 1 & 2,38 & 22 & 0 & 0 \\
\hline Total & 42 & 100,00 & & 60 & 100,00 \\
\hline \multicolumn{6}{|c|}{ Frecuencia Alélica } \\
\hline \multicolumn{3}{|c|}{ Antes del raleo genético } & \multicolumn{3}{|c|}{ Después del raleo genético } \\
\hline Alelo & Frec.Abs. & Frec.Rel. & Alelo & Frec.Abs. & Frec.Rel. \\
\hline 3 & 81 & 96,43 & 3 & 119 & 99,17 \\
\hline 2 & 3 & 3,57 & 2 & 1 & 0,83 \\
\hline Total & 84 & 100,00 & & 120 & 100,00 \\
\hline
\end{tabular}

Referencias: Frec. Abs: Frecuencia Absoluta, Frec. Rel.: Frecuencia Relativa 
El rodal de $P$. chilensis de Palampa con la aplicación de la metodología sugerida por Verga (2014) logró alcanzar condiciones mínimas admisibles de homogeneidad genética y aislamiento (Tabla 4) que le otorgarían la categoría de "Seleccionada" al poseer los umbrales de frecuencias para alelos y genotipos establecidos por la Resolución 374/14 (INASE, 2019b). Valores de frecuencia genotípica inferior al $2 \%$ para el alelo contrario a su grupo de pertenencia (alelos contrarios: 2 para el grupo de los blancos y 3 para el de los negros). El APS de $P$. chilensis, algarrobo del grupo de los blancos, de Palampa obtuvo un porcentaje de frecuencia genotípica para el genotipo $\mathrm{ADH}-\mathrm{A} 22$ de $0 \%$. Se permite una frecuencia genotípica máxima de DIEZ (10) individuos heterocigotas (ADH-A23) cada CIEN (100) individuos y en este caso obtuvo 1,67 \% por lo el APS de Prosopis chilensis de
Palampa puede ser admitido como Rodal Semillero faltando sólo los procesos administrativos exigidos por INASE.

Una vez inscripto el rodal semillero, las semillas y plantines que se obtienen del mismo podrán certificarse por Res. INASE 374/2014 (INASE, 2019b) con categoría "Seleccionada". Para el caso de P. flexuosa de Pipanaco, aún es necesario continuar con los estudios, porque no pudo ajustarse a los umbrales exigidos (Figura 1e). Cabe consignar que los rodales de Villa María ( $P$. $a l b a$ ) y San Miguel ( $P$. flexuosa) de la provincia de Córdoba fueron inscriptos como APS hasta tanto se completen los requerimientos para su inscripción como RS.

En la Tabla 5 se detallan las APS de Prosopis alba que, mediante trabajos conjuntos del BNGP e INTA han avanzado o están en proceso de ser RS.

Tabla 5. Rodales en proceso de Inscripción en INASE en la categoría de Rodal Semillero para la obtención de Semilla Seleccionada con la participación del BNGP.

Table 5. Stands be registered in INASE in the Seed Stand category to obtain Selected Seeds with the participation of the BNGP.

\begin{tabular}{lll}
\hline Especie & Localización & Razón Social \\
\hline P. alba & La Unión, Salta & Puesto Sajama \\
P. alba & Campo Durán, Salta & Comunidad Chané \\
P. alba & Villa María, Córdoba & Destefanis Osvaldo Ignacio \\
P. chilensis & Saujil, Catamarca & Renato Rossanigo. Palampa S.A \\
P. flexuosa & San Miguel, Córdoba & Sergio Cardinali e Hijos \\
P. flexuosa & Salar de Pipanaco, & El Resurgir del Algarrobal S.A. \\
& Catamarca & \\
\hline
\end{tabular}




\section{Conservación ex situ y mejoramiento}

Otras colecciones de conservación ex situ, las constituyen los ejemplares en jardines botánicos, los Rodales Semilleros Certificados y los ensayos a campo (ensayos de orígenes, progenies y procedencias, red de ensayos), en los que el BNGP ha tenido participación en mayor o menor medida, tales como los citados por Verzino et al. (2019), Lopez Lauenstein et al. (2016) y Capello (2019).

Un ejemplo de conservación ex situ es el ensayo de orígenes de especies o híbridos del género Prosopis establecido en noviembre de 2016 en el Campo Escuela de la FCAUNC en convenio con INTA, con plantines realizados con semillas procedentes de RS, APS o selección INTA por biomasa a fin de probar su adaptación y crecimiento en una región degradada del área fitogeográfica de Espinal de la provincia de Córdoba.
Los orígenes fueron ocho: RS Palampa, Catamarca ( $P$. chilensis), RS Isla Cuba, Formosa (P. alba), RS Villa María, Córdoba ( $P$. alba), APS Lomitas Blancas, Santiago del Estero (Híbridos P. alba x P. chilensis), RS Campo Durán, Salta (Prosopis alba), RS Amaicha, Tucumán (P. chilensis), RS Santiago del Estero ( $P$. alba) y Selección INTA por Biomasa (P. alba).

El crecimiento en diámetro a la base (DAB) expresados en mm y altura expresados en centímetros al año de edad se observan en la Figura 4. Se destacaron al momento, por su crecimiento en DAB, los ejemplares procedentes de los orígenes de Rodales de $P$. alba de Campo Duran, Amaicha y APS de híbridos de Santiago del Estero, mientras que en las alturas fueron homogéneas excepto los RS Palampa y Villa María que presentaron valores inferiores con respecto al resto. a)

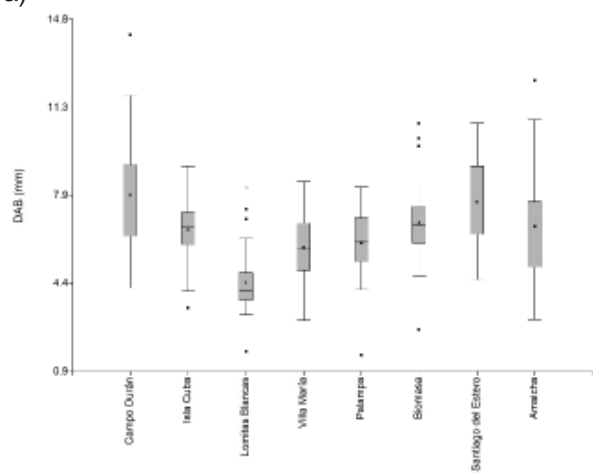

b)

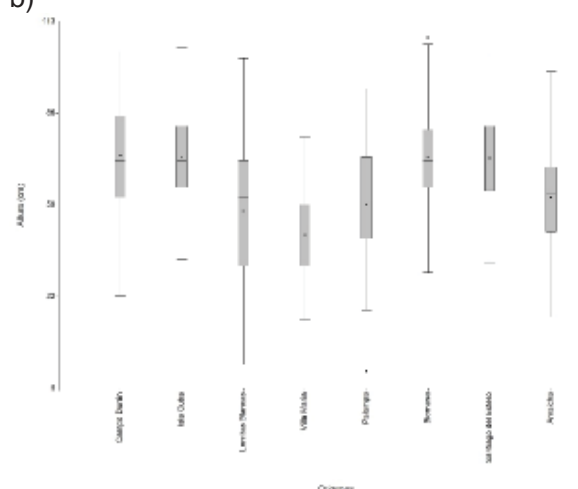

Figura 4. Crecimiento en: a) diámetro a la base (DAB) y b) altura, ambas al año de plantación del ensayo de orígenes.

Figure 4. Growth in: a) diameter at the base (DAB) and b) height, both one year after planting the origins test. 
Varios proyectos de mejoramiento genético efectuados en poblaciones de P. alba, conducidos por el INTA, se efectuaron para transformarlas en APS o RS. Dos de esas poblaciones, separados unos $30 \mathrm{~km}$ entre sí, se encuentran ubicados en bosque de ribera asociados al Río Bermejo, al oeste de la Provincia de Formosa y al sur de la Localidad de Ing. Juárez, los que se denominan Bolsa Palomo e Isla Cuba, respectivamente (Capello, 2019). Actualmente, Isla Cuba está inscriptas por el gobierno de la Provincia de Formosa en el INASE como Rodal Semillero productor de semilla de calidad seleccionada. Los materiales procedentes de las población originales generados en estos estudios (semillas de colecta masal caracterizadas morfo-genéticamente) fueron incorporados a la Colección INTA del Banco Pasivo del BNGP. En Tabla 6 se muestran detalles de la cantidad de árboles que los conformaron y también la categoría en la que se encuentran tanto para inscribirse como APS o RS según nivel de estudio alcanzado.

\section{CONCLUSIONES}

El BNGP actualmente cuenta en el Banco Pasivo con 1.650 accesiones correspondientes a 1.106 árboles de 9 especies arbóreas de Prosopis y de 8 poblaciones originales de $P$. alba que se encuentran bajo estudio para constituirse en APS o RS y que se encuentran dentro de la colección INTA del BNGP. Con la información del Banco de Datos desde 1986 y el aporte numerosas investigaciones se crean 6 RS en proceso de Inscripción en INASE en la categoría de Rodal Semillero para la obtención de Semilla Seleccionada, 14 APS inscriptas, y 17 nuevos APS en la región del Espinal aún en estudio y no inscriptos y 3 en el sur- oeste de Córdoba en proceso de inscripción. Los APS y RS logrados inscriptos se constituyen en material base de las categorías "Fuente identificada" y "Seleccionada" para abastecer a las necesidades de forestación.

Tabla 6. Materiales de P. alba incorporados a la Colección INTA del Banco Pasivo del BNGP y cantidad de ejemplares que lo constituyen.

Table 6. Materials of P.alba incorporated into the INTA Collection of the BNGP Passive Bank and number of individuals that compose each stand.

\begin{tabular}{llc}
\hline Denominación del Rodal & Población & $\begin{array}{c}\text { Cantidad de ejemplares } \\
\text { (accesiones) que lo } \\
\text { conforman }\end{array}$ \\
\hline APS2 & Galeano, Salta & 10 \\
APS4 & Bolsa Palomo, Formosa & 15 \\
RS5 & Isla Cuba, Formosa & 14 \\
APS8 & Bermejito, Chaco & 14 \\
APS9 & Chañar Bajada, Santiago del Estero & 14 \\
APS10 & Villa Angela, Chaco & 20 \\
APS11 & Animas, Santiago del Estero & 14 \\
APS12 & Presidencia de la Plaza, Chaco & 18 \\
\hline
\end{tabular}


Las estrategias de conservación y de mejoramiento tomadas desde el origen del Programa Nacional de Forestación están dando un nuevo horizonte a un género tan importante como lo es Prosopis de manera que sea posible tener material base para restaurar ecosistemas y obtener material base para programas de reforestación en Argentina. Es necesario ampliar los estudios para aumentar la cantidad de rodales semilleros a partir de las poblaciones de especies de Prosopis que se encuentran conservadas en el Banco Nacional de Germoplasma de Prosopis.

\section{AGRADECIMIENTOS}

A todos los profesionales, alumnos y productores que han contribuido al funcionamiento del Banco Nacional de Germoplasma de Prosopis, así como también a las autoridades de la FCA, UNC e INTA que apoyaron y consolidaron su crecimiento desde el momento de su creación, en el año 1985.

\section{BIBLIOGRAFÍA}

Bessega C. 1997. Estudios isoenzimáticos en especies Americanas del Género Prosopis (Leguminosae). Tesis de Licenciatura. FCE y N. Universidad de Buenos Aires, Buenos Aires, Argentina. 84 pp.

Bessega, C., Pometti, C. L., Ewens, M., Saidman, B. O., y Vilardi, J. C. 2012. Strategies for conservation for disturbed Prosopis alba (Leguminosae, Mimosoidae) forests based on mating system and pollen dispersal parameters. Tree Genetics and Genomes, 8(2), 277-288. Publicado en internet, disponible en https:// doi.org/10.1007/s11295-011-0439-6. Acceso: 13/06/2019.

Burkart A. 1976. A monograph of the genus Prosopis (Leguminosae subfam. Mimosoideae) (Part 1 and 2). Catalogue of the recognized species of Prosopis. Journal of the Arnold Arboretum 57(3): 219-249 y (4): 450-525.

Capello R. 2019. Banco de Germoplasma Forestal. Manejo de los Recursos Genéticos Forestales Nativos. Publicado en internet, disponible en https://www.formosa.gob.ar/ produccion/ recursosnaturales /bancogermoplasma. Acceso: 13/06/2019.

Castillo Ibáñez JC, Moreno Díaz GA. 2000.Semillas forestales del bosque nativo chileno. Editorial Universitaria S. A. Chile. 89 pp.

Di Rienzo JA, Casanoves F, Balzarini MG, Gonzalez L, Tablada M, Robledo CW. 2012. InfoStat versión 2012. Grupo InfoStat, FCA, Universidad Nacional de Córdoba, Argentina. URL

Eriksson G, Namkoong G, Roberds J.1995. Conservación dinámica de los recursos genéticos de los árboles forestales recursos genéticos forestales FAO, Roma. N²3. Publicado en internet, disponible en: http: //www.fao.org/3/ v8940s/V8940S02.htm. Acceso: 21/07/ 2020.

Eriksson G. 2000. Red Europea de Conservación de Recursos Genéticos de Frondosas Nobles. Invest. Agr.: Sist. Recur. For.: Fuera de Serie 2: 59-69.

FAO, IPGRI. 1994. Normas para bancos de genes. Food and Agriculture Organization, (FAO), Rome (Italy). International Plant Genetic Resources Instit., (IPGRI), Rome (Italy). 14 pp.

FAO. 1994. Código Internacional de Conducta de la FAO para la Recolección y Transferencia de Germoplasma. 20 pp. 
Eriksson G, Namkoong G, Roberds J.1995. Conservación dinámica de los recursos genéticos de los árboles forestales recursos genéticos forestales FAO, Roma. $\mathrm{N}^{\circ} 23$. Publicado en internet, disponible en: http: //www.fao.org/3/ v8940s/V8940S02.htm. Acceso: 21/07/ 2020.

FAO. 2014a. El estado de los recursos genéticos forestales en el mundo. Síntesis informativa.19 pp.

FAO. 2014b. Plan de acción mundial para la conservación y la utilización sostenible y el desarrollo de los recursos genéticos forestales p comisión de recursos genéticos para la alimentación y la agricultura.35 pp.

Ferreyra LI, Vilardi JC, López V, Verga A, Saidman BO. 2013. Genetic and morphometric markers are able to differentiate three morphotypes belonging to Section Algarobia of genus Prosopis (Leguminosae, Mimosoideae). Plant Systematics and Evolution. 1-17. Springer Vienna.

Grant V. 1981. Plant Speciation. Columbia Univ. Press. New York.

INASE. 2019a. Normativas. Normas para la Certificación, Producción, Comercialización e Importación de Semillas de Especies Forestales (Res. No 256/99). Publicado en internet, disponible en:https://www.inase.gov. ar/index.php?option=com_remository\&Itemid $=102 \&$ func $=$ select $\&$ id $=11 \&$ orderb$\mathrm{y}=2$ \&page=13. Acceso: 10/6/2019.

INASE. 2019b. Normativas. Resolución 374/14 Publicado en internet, disponible en: https://www.inase.gov.ar/index.php?option=com_remository \& Itemid $=102 \&$ func $=$ select\&id=11\&orderby=2\&page=25. Acceso: 12/6/2019.

Ipinza R, Paz Molina M, Gutiérrez B, Ortí O. 2011. Estrategia de Mejoramiento y Conservación de los Recursos Genéticos Forestales de Chile para enfrentar el Cambio Climático. Ciencia e Investigación Forestal 17 (3)359.
Joseau, J., Verga, A., y Díaz, M. 2005. Los recursos genéticos de Prosopis. IDIA XXI. Revista de Información sobre Investigación y Desarrollo Agropecuario.(Jul 2005). a. 5 (8), 207-211.

Joseau, M. J. 2006. Caracterización morfológica y genética de poblaciones del género Prosopis del Chaco semiárido del norte de Córdoba y sur de Santiago del Estero. Tesis para optar al grado de Doctora en Ciencias Agropecuarias. Escuela para Graduados de la Facultad de Ciencias Agropecuarias. Univeridad Nacional de Córdoba. 151 pp.

Joseau MJ, Verzino G, Hernández R, Meehan A 2012. Enriquecimiento con Prosopis alba Griseb., P. chilensis (Mol.) Stuntz y P. nigra (Griseb.) Hieron. en bosques degradados del Espinal de Córdoba. V Jornadas Integradas de Investigación y Extensión. 31 de Octubre y 1 de Noviembre de 2012. En CD.

Joseau MJ, Aráoz SD, Hernández R. 2013a. La semilla. En: Conservación de recursos forestales nativos de Argentina. El cultivo de plantas leñosas en vivero y a campo. Joseau MJ, Conles MY, Verzino GE, editores. Editorial Brujas. III: 53-90.

Joseau MJ, Verga AR, Díaz M del P, Julio NB. 2013b. Morphological diversity of populations of the Genus Prosopis in the Semiarid Chaco of Northern Cordoba and Southern Santiago Del Ester”. American Journal of Plant Sciences.4 (11) 2092 :2111.

López Lauenstein D, Fernández M, Verga A. 2013. Drought stress tolerance of Prosopis chilensis and Prosopis flexuosa species and their hybrids. Trees-structure and function. $27: 285-296$

López Lauenstein D, Vega C, Luna. C, Sagadín M, Melchiorre M, Pozzi E, Salto C, Obeschelp J, Torales S, Pompono F, Kess S, Chávez Díaz L, Gomez C, Verga A. 2016. Sub- 
M. J. Joseau et al.

programa Prosopis. En: Domesticación y mejoramiento de especies forestales. $201 \mathrm{pp}$. Publicado en internet, disponible en http:// forestoindustria.magyp.gob.ar/archivos/biblioteca-forestal/domesticacion-y-mejoramiento-de-especies-forestales.pdf. Acceso: 12/6/2019.

Mallén Rivera C. 2014. Recursos Genéticos Forestales. Nota del editor. Revista Mexicana de Ciencias Forestales. 5(22): 4-9.

Marcó MA, Gallo LA, Verga AR. 2016. Domesticación y mejoramiento de especies forestales. 201 pp. Publicado en internet, disponible en http://forestoindustria.magyp.gob. ar/archivos/biblioteca-forestal/domesticación-y-mejoramiento-de-especies-forestales. pdf.Acceso:12/6/2019.

Namkoong G.1976. A multiple index selection strategy. Silvae Genet. 25:199-201.

Namkoong G. 1984. A control concept of gene conservation. Silvae Genet. 33:160- 163.

Rohlf FJ. 2000.NTSYS-pc numerical taxonomy and multivariate analysis system version 2.1 manual. Applied Biostatistics, New York, NY. 43 pp.

Palacios R, Agulló MA, Mom MP, Torregrosa S, Picca P. 1988. Especies del Género. Taxonomía. En: Prosopis en Argentina. Documento preliminar elaborado para el Primer Taller Internacional sobre Recurso Genético y Conservación de Germoplasma en Prosopis. FAO, FCA-UNC y FCEy N-UBA. Cosquín, Córdoba, Argentina.Pp.15-96.

Pasiecznik NM, Felker P, Harris PJC, Harsh LN, Cruz G, Tewari JC, Cadoret K, Maldonado LJ. 2001. The Prosopis juliflora-Prosopis pallida Complex: A Monograph. HDRA. Coventry U.K. 162 pp.

Rocha L. 2018. Subió la deforestación y preocupa la baja partida a la protección de bosques en el nuevo Presupuesto- Infobae- Sociedad. Publicado en internet, disponible en https:// www.infobae.com/sociedad/2018/10/28/subio-la-deforestacion-y-preocupa-la-baja-partida-a-la-proteccion-de-bosques-en-el-nuevo-presupuesto/. Acceso: 12/6/2019.

SAyDS (Secretaría de Ambiente y Desarrollo Sustentable). 2003. Resolución 91/03. Argentina y Política Ambiental. Secretaría de Ambiente y Desarrollo Sustentable. Argentina. Publicada en internet, disponible en http://www.ecofield.net/Legales/Pol_ambiental/res91-03_SAyDS.html\#arriba. Acceso: $12 / 6 / 2019$.

Saidman BO. 1986. Isoenzymatic studies of alcohol dehydrogenase and glutamate oxalacetate transaminase in four South American species of Prosopis and their natural hybrids. Silvae Genetica 35: 3-10.

Salazar E, León P, Rosas M, Muñoz C. 2006. Estado de la conservación ex-situ de los recursos fitogenéticos cultivados y silvestres en Chile. Instituto de Investigaciones Agropecuarias. Boletín INIA N 156 . Santiago, Chile. 180 pp.

Verga, A. 2000. Hojas. Programa para la medición de caracteres morfológicos de hojas. INTA. IFFVE. [software].

Verga AR. 1995. Genetic study of Prosopis chilensis y Prosopis flexuosa(Mimosaceae) in the dry Chaco of Argentina. Tesis Doctoral. Göttingen Researh Notes in Forest Genetics. Abteilung für Forstgenetik und Forstpflanzensüchtung der Universität Göttingen. Göttingen, Alemania. 96 pp.

Verga, A. 2005. Recolección de material de algarrobo en el Chaco húmedo y subhúmedo. SAGPyA Forestal, 34, 2-7.

Verga AR. 2010. Hojas. Programa para la medición de caracteres morfológicos de hojas. INTA. IFFVE. Córdoba. Argentina

Verga, A. 2014. Rodales semilleros de Prosopis a partir del bosque nativo. Quebracho.19 $(1,2): 125-138$ 
Verga A, López Lauenstein D, López C, Navall M,Joseau J, Gómez C, Royo O, DeganoW, MarcóM. 2009. “Caracterización morfológica de los algarrobos (Prosopis sp.) en las regiones fitogeográficas Chaqueña y Espinal norte de Argentina”. Quebracho 17 (1,2): 31-40.

Verga A, López C, Naval M, Joseau J, Gómez C, Royo O, Degano W, Marcó M. 2014. Caracterización morfológica, distribución geográfica y estimación de nichos ecológicos de algarrobos (Prosopis sp.) en las regiones fitogeográficas Chaqueña y Espinal norte de Argentina. http://redaf.org.ar/wp-content/ uploads/2008/02/Distribucion-geografica-Prosopis.pdf. Acceso:12/6/2019.

Verzino GE, Joseau MJ. 2005. Uso, mejoramiento genético, y transferencia del germoplasma de Prosopis. En: Conservación de recursos forestales nativos en Argentina. El Banco Nacional de Germoplasma de Prosopis. Verzino GE, Joseau MJ, editores. Córdoba, Argentina. Pp. 107-117.

Verzino G. y Joseau MJ. 2013. Plantaciones forestales con especies nativas de Argentina en la región central del país. En: Conservación de recursos forestales nativos de Argentina. El cultivo de plantas leñosas en vivero y a campo. Editores: Joseau MJ., Conles MY. y Verzino G E. Editorial Brujas. X: 235-275.
Verzino GE, Frassoni JE, Joseau MJ, Clausen G, Navarro C. 2019. Conservación ex situ, circa situ e in situ realizada por el Banco Nacional de Germoplasma de Prosopis, Córdoba, Argentina. Revista Nexo 7 (1) 42:52

Verzino GE., Joseau MJ., Frassoni JE. 2020. Elaboración de un protocolo de gestión para bancos de germoplasma de especies leñosas de Argentina con semillas ortodoxas. El protocolo del Banco Nacional de Germoplasma de Prosopis (BNGP). Primera Parte. Nexo Agropecuario. 8(1):60-70.

Zárate MH, Fernández R, Tato Vázquez C, Acosta N, Pérez V, Kees S, Arce L. 2016. Avances en la silvicultura del algarrobo blanco. MAGyP- UNAF- INTA. 33 pp. Publicada en internet, disponible en: https://inta.gob. ar/ sites/default/files/avances_prosopis.pdf. Acceso:12/6/2019 